\title{
Consultas de urgencia posteriores al terremoto del 27 de febrero 2010, en el hospital de Cauquenes
}

MAURICIO CONTRERAS ${ }^{(1)}$, JUAN ABARZA ${ }^{(1)}$, FRANCISCA ARAYA ${ }^{(1)}$, STEPHANIE DUPONT $^{(2)}$, CEDRIC DANNIAU $^{(2)}$, CONSTANZA CASTELLI ${ }^{(3)}$ y PABLO GARRIDO ${ }^{(1)}$

\section{RESUMEN}

Se realizó un estudio descriptivo transversal según frecuencia de consultas en el servicio de urgencia del Hospital San Juan de Dios de Cauquenes, tipo de lesiones, y apoyo utilizado, para caracterizar la morbilidad asociada al sismo ocurrido entre la Quinta y Novena regiones de Chile, el 27 de febrero de 2010.

El Universo fue constituido por todos los casos atendidos posteriormente al desastre en dicho servicio, y la información fue recogida a partir de los sistemas de estadísticas continuas de este mismo establecimiento.

Fallecieron en la comuna de Cauquenes, 15 personas el día mismo del terremoto, con una tasa de 3,5 por 10.000 habitantes; se registraron 1360 consultas en los primeros 10 días, con predominio de traumatismos superficiales, heridas y fracturas de los miembros.

Los resultados obtenidos son de utilidad en el logro de un enfoque más racional para la atención, la prevención y el auxilio ante un desastre en regiones con similares características.

Palabras clave: terremoto 27-02-2010, consultas urgencia, trauma.

\section{ABSTRACT}

POST EARTHQUAKE EMERGENCY CONSULTATIONS AT THE CAUQUENES HOSPITAL, FEBRUARY 27, 2010

A descriptive, cross sectional study was carried out to characterize the morbidity associated with the earthquake that occurred on February 27, 2010, affecting the Fifth to Ninth administrative regions in Chile. The frequency of consultation, type of injury, and support used in the emergency department of the San Juan de Dios Hospital of Cauquenes were analyzed. The universe consisted in all the cases seen after the disaster at that emergency department, and the information was collected from the current statistics systems at the hospital. In Cauquenes County, there were 15 deaths the day of the earthquake, a mortality rate of 3.5 per 10,000 residents. 1360 consultations were registered in the first 10 days, mainly superficial injuries, wounds and fractures. The obtained results are useful for achieving a more rational approach to care, prevention and aid in a disaster situation with similar characteristics.

Keywords: 02-27-2010 earthquake, emergency consultations, trauma.

(1) Hospital San Juan de Dios de Cauquenes. Calle Montt sin número. Cauquenes. VII Región. Chile. mitocontreras@yahoo.com

(2) Internos Facultad de Medicina. Université Catholique de Louvain-la-Neuve. Bélgica.

(3) Alumna Facultad de Medicina. Universidad de Valparaíso. V Región. Chile. 


\section{INTRODUCCIÓN}

A las 3h34'17" de la mañana del día 27 de febrero del 2010, un terremoto grado 8.8 en la escala de Richter con epicentro a $63 \mathrm{Km}$. de Cauquenes, afectó desde la Quinta a la Novena regiones de Chile. Veinticinco minutos después, fue seguido por un tsunami, provocando una gran devastación principalmente en la zona costera.

La Séptima Región, lugar del epicentro, tiene una dotación de 13 hospitales ${ }^{1}$ (Figura 1); consta de una población de 1.007.831 habitantes ${ }^{2-3} \mathrm{y}$, a su vez, la provincia de Cauquenes tiene una población de 60.103 habitantes, y la comuna del mismo nombre $42.796^{4}$.

El terremoto destruyó los hospitales de mayor complejidad pertenecientes a Talca y Curicó (Tipo 1), y los de mediana complejidad de Parral y Cauquenes (Tipo 3). Este último particularmente, se vio severamente afectado por el sismo, resultando en la pérdida de las salas de hospitalización correspondientes a pediatría, maternidad, medicina interna, cirugía, radiología y pabellones. Sin embargo, por su cercanía al epicentro además de prestar servicios a la comuna, fue un lugar central para la atención de los pacientes derivados desde Pelluhue, Curanipe y Chanco. Fallecieron en la comuna de Cauquenes 15 personas el día mismo del terremoto ${ }^{2-3}$, con una tasa de 3,5 por 10.000 habitantes.

Es teóricamente esperable que en las horas inmediatamente siguientes a una catástrofe de estas características, se registre un aumento significativo en el número de consultas de tipo traumatológico ${ }^{5-6}$. Posteriormente, debido a las condiciones higiénicas y ambientales derivadas de la falta de acceso a agua potable y a alcantarillado y la pérdida de viviendas, sería esperable un incremento en las patologías digestivas de tipo infeccioso y en las enfermedades respiratorias con respecto a la situación pre terremoto ${ }^{6-7}$.

Se realizó un análisis de las consultas efectuadas en el servicio de urgencia en los 10 días posteriores al sismo, considerando para esto el sexo y tipo de patología, y contrastando estas cifras con los datos correspondientes a los 10 días previos al terremoto.

Como un segundo objetivo, se intentó analizar con mayor profundidad los subtipos de lesiones traumatológicas, su evolución, y los
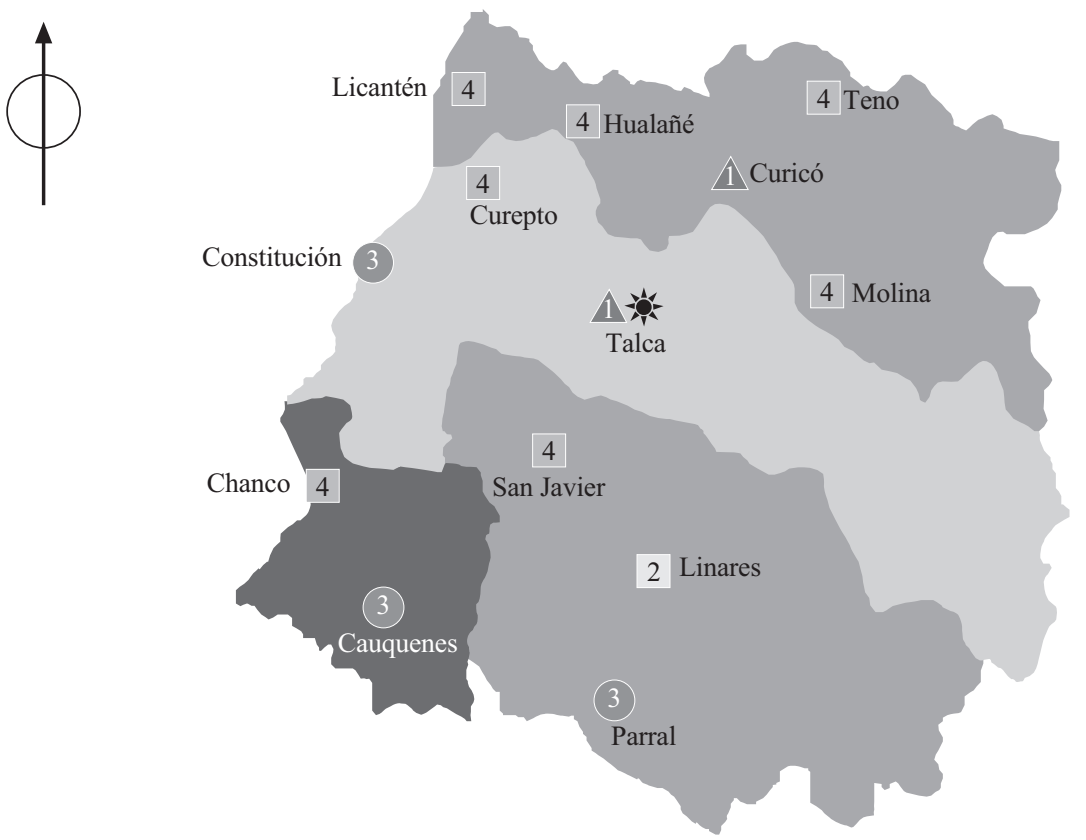

Simbología

1 Hospital tipo 1

2 Hospital tipo 2

3 Hospital tipo 3

4 Hospital tipo 4

猔- Dirección SSM

Figura 1. Red de hospitales, VII Región del Maule 


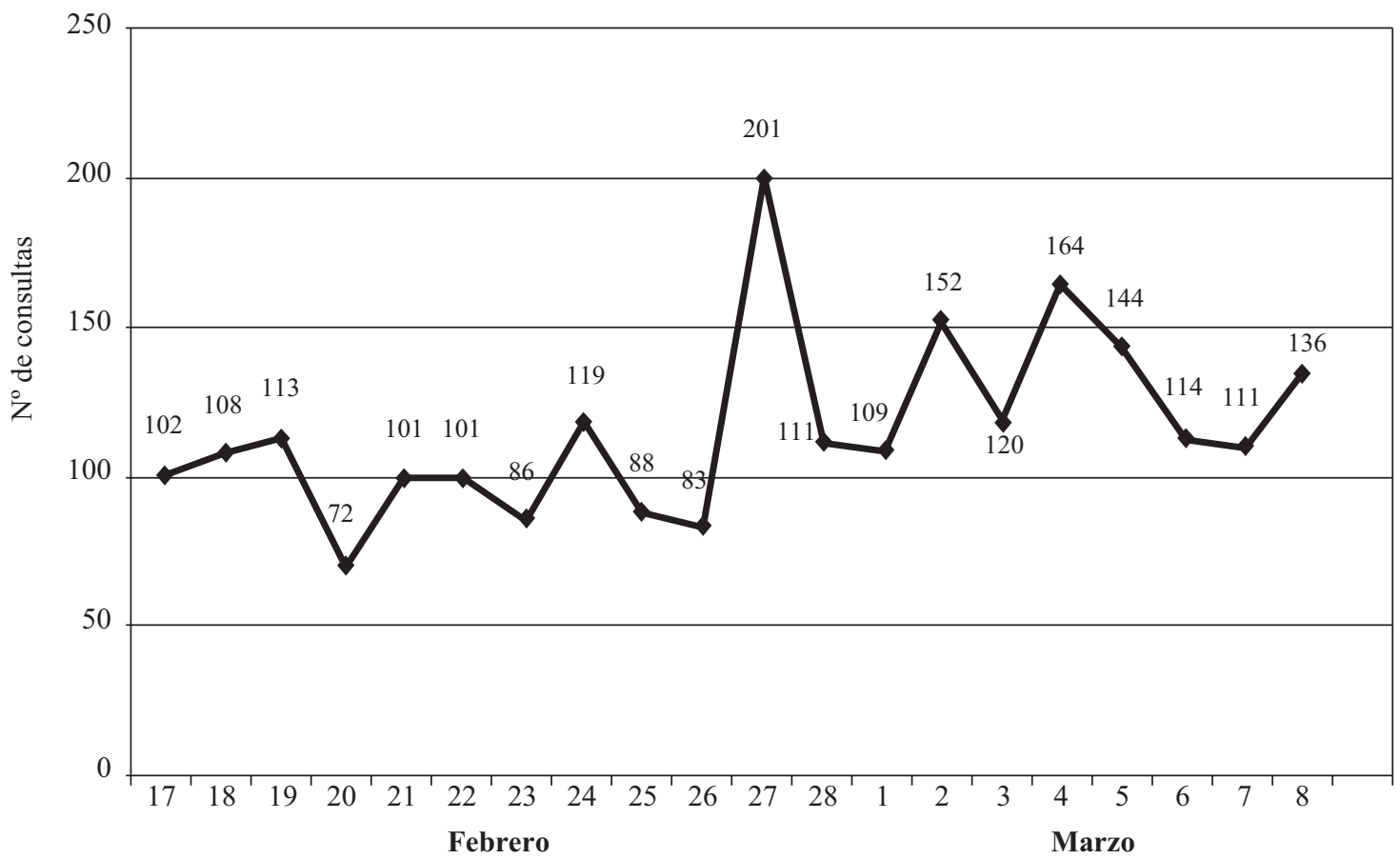

Figura 2. Atenciones de urgencia 10 días antes y 10 días después del terremoto del 27 de febrero del 2010.

insumos (curación, sutura, inmovilización) utilizados en su tratamiento, permitiendo así obtener datos sobre el tipo y cantidad de ayuda externa y reservas intrahospitalarias requeridas para mantener la efectividad de un hospital en una situación aguda de similares características.

\section{MATERIAL Y MÉTODO}

Se realizó un estudio epidemiológico descriptivo transversal. Para la caracterización de la morbilidad, el universo estuvo constituido por todas las personas atendidas en el servicio de urgencias del hospital San Juan de Dios de Cauquenes durante los 10 días anteriores y los 10 días posteriores al terremoto del 27 de febrero de 2010 (esta fecha es considerada en el estudio como primer día posterremoto, ya que el desastre tuvo lugar en la madrugada de éste). La información fue recogida por los sistemas de estadísticas continuas de este mismo hospital.

Es importante consignar que la atención primaria de salud de la comuna no operó durante la primera semana posterremoto,
El diagnóstico de las lesiones fue codificado según la clasificación internacional de enfermedades (CIE - 10), y los datos se introdujeron en una base de datos creada en una computadora personal. Los resultados se expresaron en porcentajes y números absolutos.

Los datos se analizaron de la manera siguiente:

1. Se compararó la cantidad diaria de consultas en urgencia los 10 días previos al terremoto con los 10 días posteriores.

2. Se comparó la proporción hombre-mujer de los pacientes atendidos 10 días previos al terremoto con los 10 días posteriores.

3. Se comparó el tipo de patología diagnosticada entre los 10 días previos al terremoto y los 10 días posteriores (se utiliza la clasificación CIE10), clasificándose en 6 categorías : Traumatológica (traumatismos, envenenamientos y algunos otros como consecuencia de causa externa); enfermedades del aparato digestivo; enfermedades del aparato respiratorio; enfermedades del aparato circulatorio (cardiovasculares); neuropsiquiátrica (enfermedades del 
sistema nervioso y trastornos mentales y del comportamiento) y otras causas(engloba toda patología que no corresponda a las categorías mencionadas anteriores)

4. Analizar desde el punto de vista traumatológico la proporción de los diferentes tipos de lesiones presentadas en los 10 días posterremoto: heridas, trauma de tórax, trauma de cráneo, trauma de columna, luxaciones y contusiones de miembros, fracturas de miembros.

5. Se analizó la evolución en los 10 primeros días posterremoto de las lesiones traumáticas predominantes: fracturas, luxaciones, heridas, contusiones.

6. Evaluar en cantidad y proporción de insumos utilizados para el tratamiento de las lesiones traumáticas en los 10 primeros días posterremoto: curación, suturas, inmovilización.

\section{RESULTADOS}

El número de consultas atendidas en urgencia tuvo un peak el día 27 de febrero, con una media de consultas de 97,3 por día antes del terremoto, 201 el día 27 de febrero 2010, y una media de 116,1 por día después del terremoto. (Figura 2)

En relación al sexo de los pacientes atendidos
10 días antes del terremoto, 53,34\% corresponde a mujeres y $46,66 \%$ hombres. Con respecto a los pacientes atendidos 10 días después del terremoto $52,4 \%$ fueron mujeres y $47,6 \%$ hombres. (Figura 3).

En la totalidad de las patologías diagnosticadas en los 10 días posterremoto comparadas con los 10 días anteriores a éste, disminuye la cantidad (en números) de enfermedades respiratorias ( $\mathrm{x}$ $0,76)$, digestivas $(\mathrm{x} 0,66)$ y cardiovasculares $(\mathrm{x}$ $0,96)$, mientras que aumenta la cantidad de traumatismos (x 2,93) y enfermedades neuropsiquiátricas $(\mathrm{x} 2,6)$.

Porcentualmente baja la cantidad relativa de enfermedades respiratorias (x 0,59$)$, digestivas ( $\mathrm{x}$ $0,5)$, cardiovasculares $(\mathrm{x} 0,66)$; y aumenta la cantidad relativa de traumatismos $(\mathrm{x} 2,05)$ y enfermedades neuropsiquiátricas (x 2). (Figuras 4 y 5)

De los traumatismos llegados en los 10 días después del terremoto, se diagnostican en orden de frecuencia: heridas (43\%), luxaciones y contusiones de miembros $(27 \%)$, trauma de cráneo $(11 \%)$, fracturas de miembros $(8 \%)$, trauma de columna ( $8 \%)$, y trauma de tórax (3\%). (Figura 6)

Lo más frecuente en los primeros días después del terremoto fueron las heridas y contusiones. Se diagnosticaron 65 contusiones

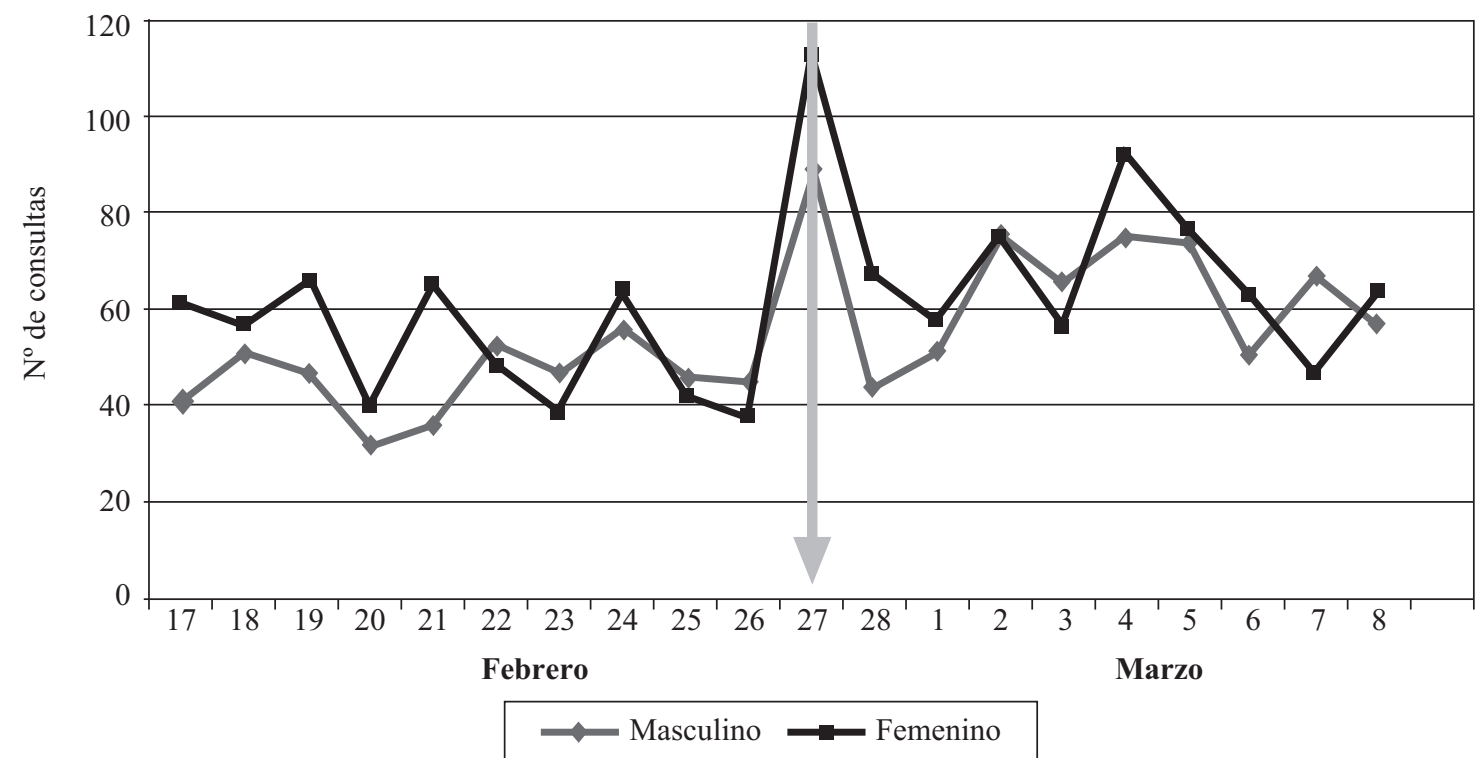

Figura 3. Relación masculino-femenino antes y después del terremoto. 
Tabla 1: Número de consultas por tipo de patología pre y posterremoto.

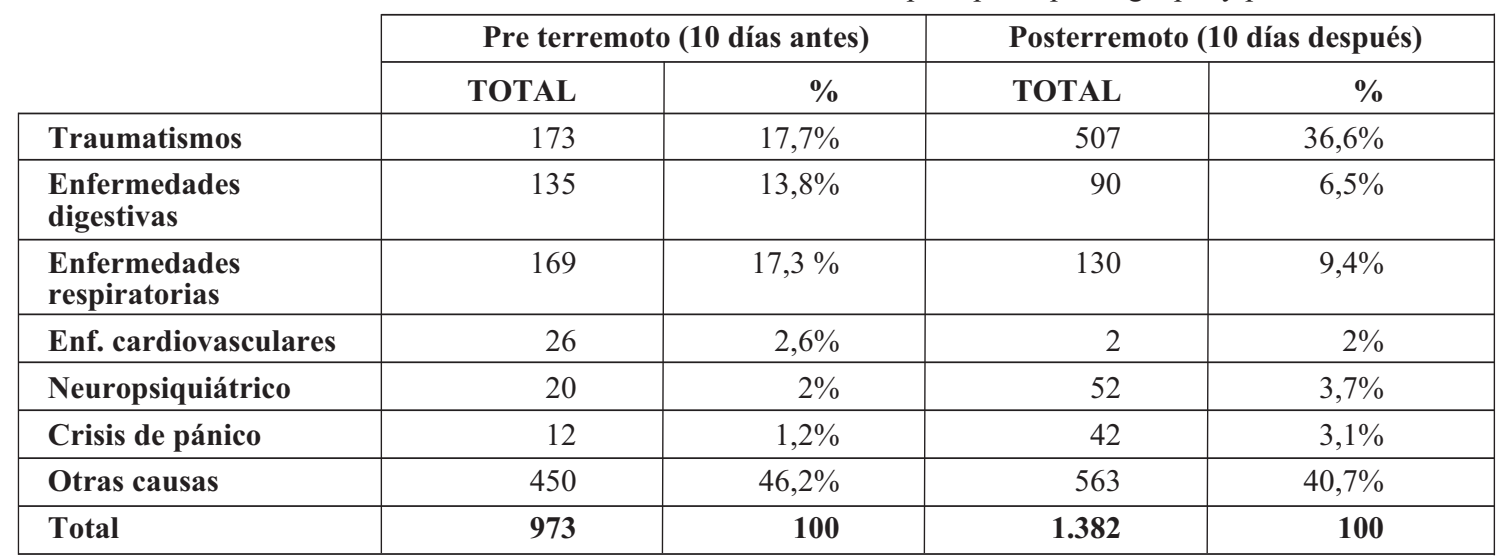

y 57 heridas el primer día y 27 contusiones junto a 26 heridas, el segundo. Se observó una elevación (22) en la cantidad de pacientes con contusiones el día 6 posterremoto y de pacientes con heridas (27) el día 7 posterremoto.

En los 10 días que siguen al sismo, las fracturas y luxaciones diagnosticadas diariamente se mantienen bajo 10, y se observa un peak en el diagnostico de éstas el día 4 posterremoto, con 8 fracturas y 7 luxaciones. Hay que destacar que el servicio de radiología empezó a funcionar nuevamente 4 días después del terremoto. (Figura 7)

Se puede observar que la cantidad de insumos utilizados aumentó notablemente en el período posterremoto. El uso de material de curación aumento en un $400 \%$, el de sutura en $300 \%$ y el de inmovilización en $760 \%$. (Figura 8 )

Cabe señalar que el aumento de las heridas se asoció también a un gran incremento en el uso de antibióticos para evitar posibles infecciones, sin embargo, estos datos no son analizables debido a que no existe un registro oficial.

\section{DISCUSIÓN}

1. El número de consultas aumentó con el terremoto. En comparación con la media de
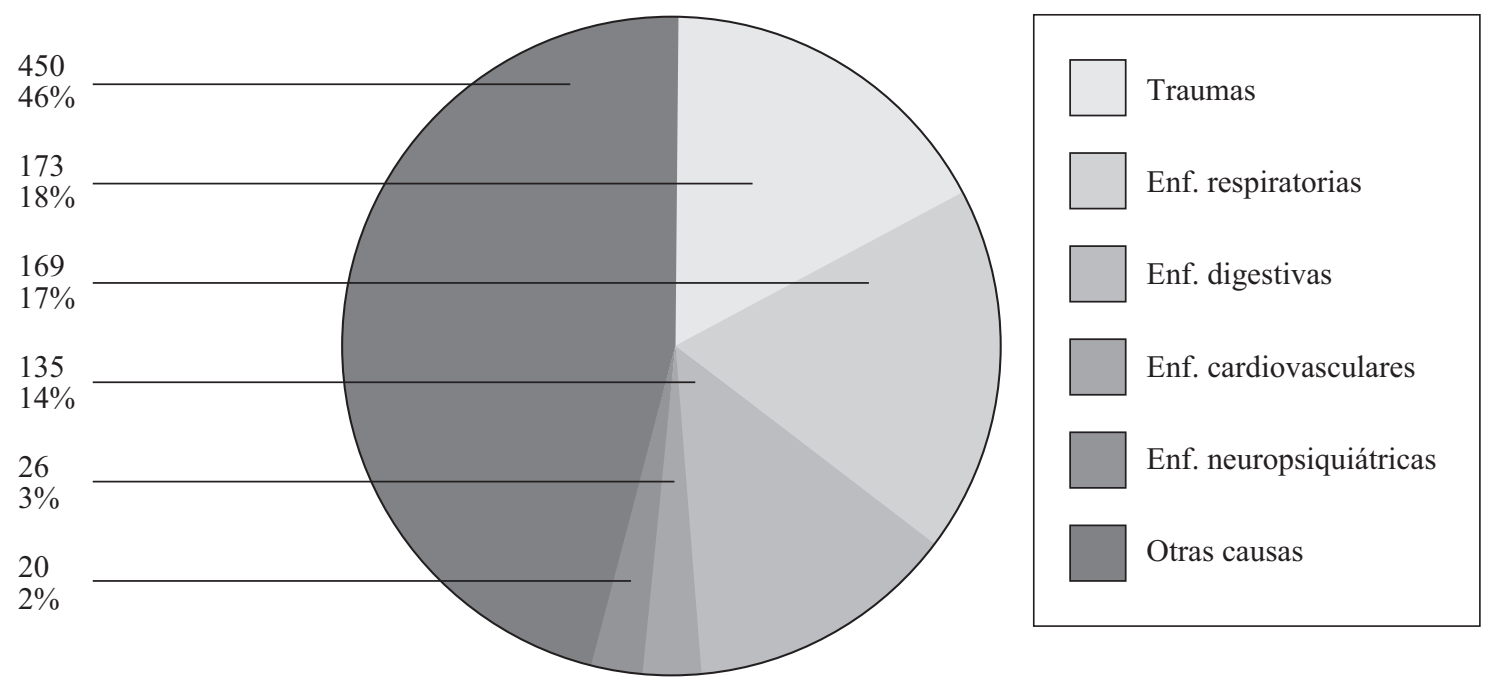

Figura 4. Tipo de consulta pre terremoto 

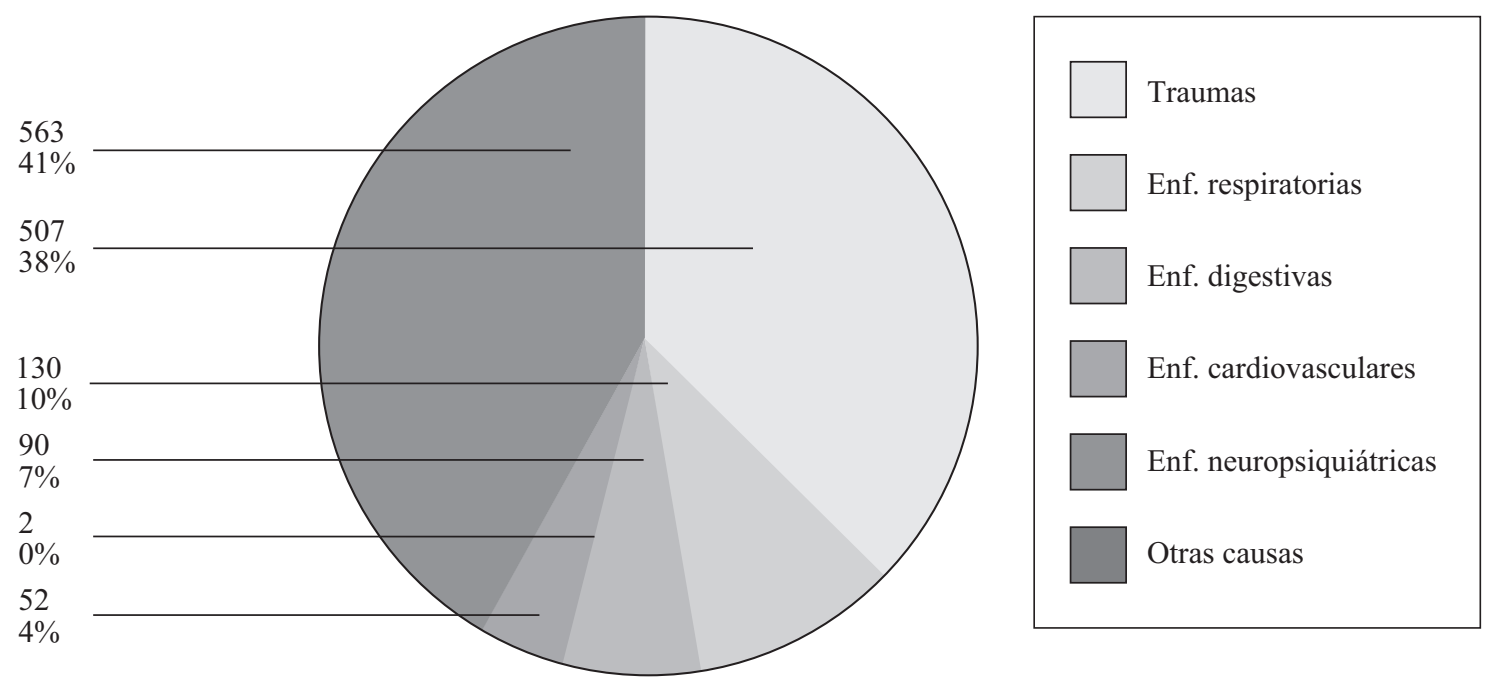

Figura 5. Tipo de consulta posterremoto

los 10 días previos, la cantidad de consultas creció a más del doble el día del sismo, aumentando en promedio un $19.32 \%$ en el periodo de los 10 días posterremoto. Esto podría explicarse por un aumento en el número de pacientes heridos y a los problemas de salud mental que se evidenciaron inmediatamente post evento. Sin embargo, también se podría asociar a que el servicio de urgencia del Hospital base de Cauquenes fue el único centro de atención de salud que funcionó el 27 de febrero y la semana posterior al terremoto, ya que los consultorios y el servicio de atención de urgencia (SAPU) de la ciudad quedaron aislados como consecuencia de la caída de un puente que los conectaba con el resto de la ciudad.

2. El porcentaje de hombres y mujeres que consultó en el servicio de urgencias no presentó cambios importantes, con respecto a los días previos al sismo.

3. Los traumatismos constituyeron una gran parte de las patologías presentadas en los 10 días después del terremoto. Igualmente se evidenció aumento de la patología neurosiquiátrica, lo que es coincidente con la literatura ${ }^{5}$, duplicándose estos casos en comparación a los días anteriores al terremoto. Las consultas por enfermedades respiratorias y digestivas, por el contrario, disminuyeron después del sismo.

4. Los traumatismos analizados fueron principalmente heridas, contusiones y fracturas. Heridas y contusiones constituyeron la mayoría de las consultas por trauma, principalmente durante los 2 primeros días posterremoto. El peak de diagnostico de fracturas y luxaciones se corresponde con el día en que se restableció el servicio de radiología.

5. Aumentó de manera significativa la cantidad de insumos utilizados para curar, suturar e inmovilizar pacientes.

\section{CONCLUSIONES}

La magnitud de un terremoto de $8.8^{\circ}$ Richter, como fue el del día 27 de febrero del 2010, produjo tal daño que la Séptima y Octava regiones de Chile fueron declaradas zona de catástrofe. Por ello, debe suponerse que las consecuencias en la salud de la población afectada son importantes.

Tras un análisis de las personas atendidas en el servicio de urgencia del hospital San Juan de Dios de Cauquenes en los 10 días después del terremoto, se puede concluir que:

1. Es esperable un aumento de la cantidad de consultas en urgencias después de una catástrofe natural de este tipo, tal como se 


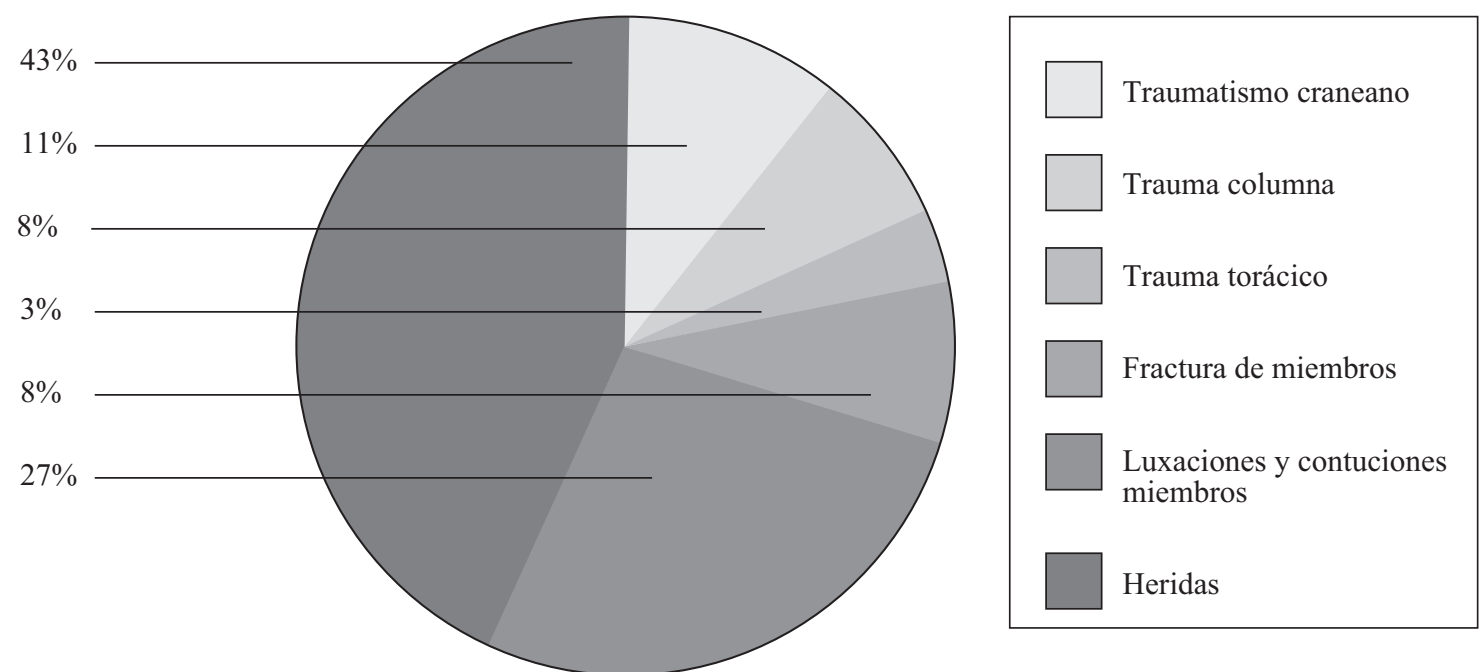

Figura 6. Tipo de lesiones traumáticas posterremoto

evidenció en la estadística, principalmente durante las primeras horas post sismo ${ }^{8}$.

2. El terremoto no cambió de manera significativa la proporción hombre-mujer, de los pacientes que consultaron en el servicio de urgencias antes, el día mismo ni después del terremoto.

3. Como se supuso al inicio, las patologías de tipo traumático constituyeron una parte considerable de las consultas en urgencias producidas por el terremoto a causa de los derrumbes y posiblemente de las labores de recolección de escombros y de reparación de viviendas y de habilitación de viviendas de emergencia realizadas por los ciudadanos en sus hogares. Se debe notar que las enfermedades digestivas (por falta de agua potable) y enfermedades respiratorias (por falta de techo y vida en carpa) no aumentaron entre las patologías diagnosticadas durante los 10 días posterremoto. Esto podría atribuirse a que durante estos días el tiempo

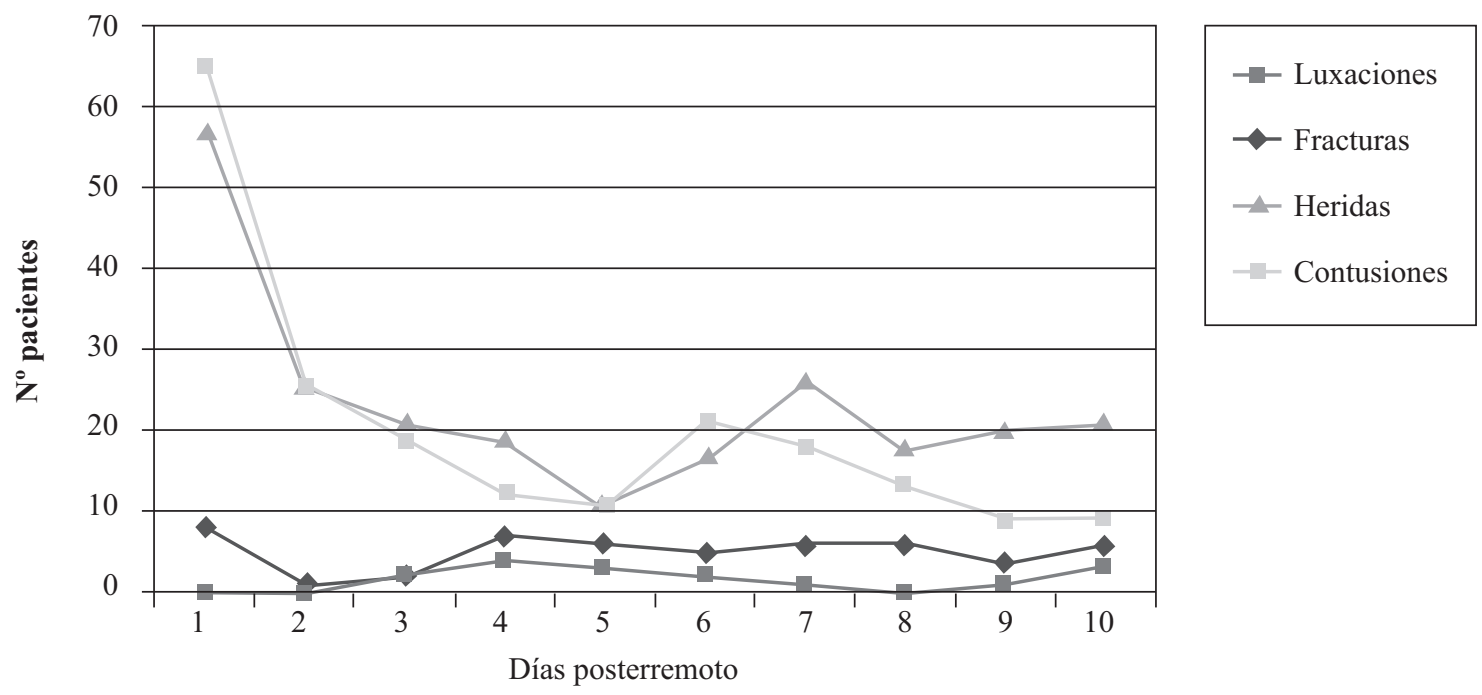

Figura 7. Consultas traumatológicas a consecuencia del terremoto. 
fue especialmente favorable y seco, y al hecho de que se distribuyó agua en camiones aljibes a la población de la provincia, lo que sería un factor de alta relevancia ${ }^{7}$. También podría explicarse por el período de tiempo considerado en el estudio (10 días posterremoto)

4. Se evidenció que un hospital debe estar preparado ante un caso de catástrofe de este tipo para atender traumatismos, sobre todo heridas y contusiones en los primeros días. El hecho de que fracturas y luxaciones se confirmaran con un retraso de 4 días después del terremoto puede atribuirse al daño producido en el servicio de radiología, mientras que la mayor frecuencia de heridas en comparación con otras patologías es fácilmente atribuible a los derrumbes y labores de reparación de las viviendas.

5. Estos datos podrían ser extrapolables a otras situaciones de similares características, por lo que puede deducirse que un hospital debe mantenerse atento a la cantidad de insumos en reserva, pues en condiciones de desastre pueden aumentar hasta 7 veces el consumo diario necesario. Entonces, es importante que un hospital conozca su capacidad de respuesta a la catástrofe, para permitir una ayuda externa rápida y adecuada.

\section{REFERENCIAS}

1. MINISTERIO DE SALUD. Chile. Servicio de Salud del Maule. Demografía y Hospitales en Región del Maule. Disponible en www.minsal.cl

2. MINISTERIO DEL INTERIOR. Chile. Resultado terremoto 27 febrero 2010, Listado oficial de personas fallecidas, disponible en http://www.interior.gov.cl/.

3. MINISTERIO DE SALUD. Chile. DEIS, Hospitales Región del Maule. www.Minsal.cl.

4. MINISTERIO DE INTERIOR. Chile. Subsecretaría

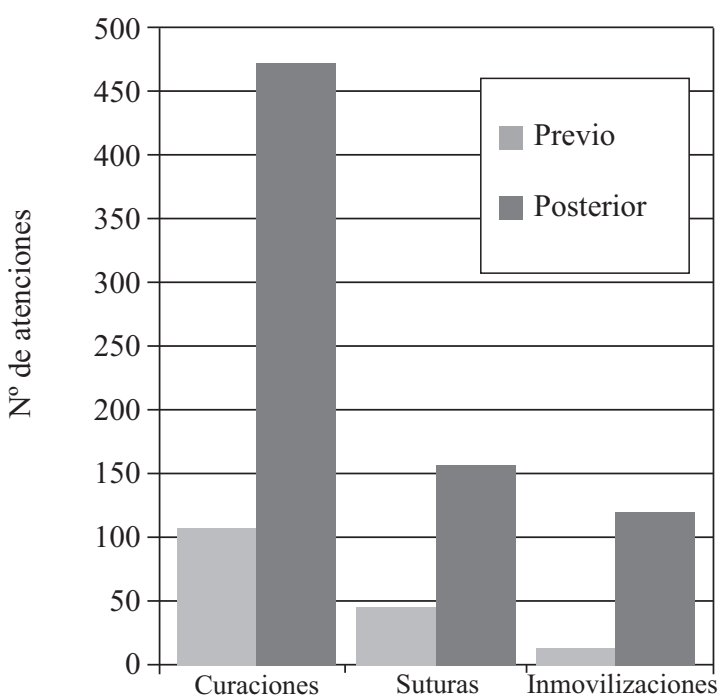

Figura 8. Procedimiento asosiados a trauma.

de Desarrollo Regional. Gobierno Regional del Maule. Provincia de Cauquenes. Población de la región del Maule y la provincia de Cauquenes, disponible en www.subdere.org.cl :

5. VENTURINI G ; ACEVEDO S; ALBORNOZ V; ARINOVICHE, M; ET AL . Estructura de la demanda de un servicio de urgencia en situación de catástrofe /Cuad. méd.-soc. (Santiago de Chile);27(1):33-8, mar. 1986.

6. RODRÍGUEZ SALVÁ A. Estrategia de atención primaria y situación de salud y saneamiento básico en condiciones de desastre tesis de maestría en salud ambiental. Ciudad de La Habana:INHEM; 2000

7. ORGANIZACIÓN PANAMERICANA DE LA SALUD. Los desastres naturales y la protección de la salud. Washington, D.C.: OPS, C2000.xi, 131 p. (Publicación Científica, 575).

8. ARMANDO RODRÍGUEZ SALVÁ Y BLANCA TERRY BERRO. Atenciones médicas urgentes y lesiones fatales como consecuencia del terremoto en Armenia. Colombia Instituto Nacional de Higiene. Epidemiología y Microbiología (INHEM).

Recepción: 22 de abril de 2010 Aprobación: 26 de agosto de 2010

Usted puede comentar éste y otros artículos publicados en la Revista Chilena de Salud Pública, enviando un correo electrónico a revistasp@med.uchile.cl 